\title{
ON THE ZEROS OF THE RIEMANN ZETA-FUNCTION
}

\author{
BRUCE C. BERNDT
}

\section{Introduction. If}

$$
R(s)=\pi^{-8 / 2} \Gamma(s / 2) \zeta(s),
$$

the functional equation for the Riemann zeta-function $\zeta(s)$ is given by $R(s)=R(1-s)$. Berlowitz [1] has recently shown that if $0<\lambda<1$, then both $\operatorname{Re} R(\lambda+i t)$ and Im $R(\lambda+i t)$ vanish infinitely often. In this note we shall give an improvement on this result.

Let $N_{R}(\lambda, T)$ denote the number of zeros for $\operatorname{Re} R(\lambda+i t)$ on $0<t<T$. Similarly, $N_{I}(\lambda, T)$ denotes the number of zeros for Im $R(\lambda+i t)$ on $0<t<T$.

THEOREM. If $0<\lambda<1$, then

$$
N_{R}(\lambda, T)>A T
$$

and

$$
N_{I}(\lambda, T)>A T
$$

where $A=A(\lambda)$.

Here and elsewhere $A, A_{1}$ and $A_{2}$ denote positive constants and $K_{1}$ and $K_{2}$ complex constants, none of which is necessarily the same with each occurrence.

For $\lambda=\frac{1}{2}$, the result follows from a famous theorem of Hardy and Littlewood [2, p. 222]. In fact, we use their method [2, pp. 222-226] in our proof.

2. Proof of the theorem. We prove (1.1) for the case $0<\lambda<\frac{1}{2}$. The proof for $\frac{1}{2}<\lambda<1$ will then follow from the functional equation $R(s)=R(1-s)$.

From [1] we have

$\frac{1}{2 \pi} \int_{-\infty}^{\infty} \operatorname{Re} R(\lambda+i t) e^{i \xi t} d t=\left(e^{-\lambda \xi}+e^{-(1-\lambda) \xi}\right) \psi\left(e^{-2 \xi}\right)-\frac{1}{2}\left(e^{\lambda \xi}+e^{(1-\lambda) \xi}\right)$,

where $\psi(x)=\sum_{n=1}^{\infty} \exp \left(-n^{2} \pi x\right)$ and $\operatorname{Re} e^{-2 \xi}>0$. Putting

$$
\xi=-i(\pi / 4-\delta / 2)-y, \quad \delta>0,
$$

we see that

Received by the editors September 5, 1968. 


$$
F(t)=\frac{1}{(2 \pi)^{1 / 2}} \operatorname{Re} R(\lambda+i t) e^{(\pi / 4-\delta / 2) t}
$$

and

$$
\begin{aligned}
f(y)= & \left(e^{\lambda i(i(\pi / 4-\delta / 2)+y\}}+e^{(1-\lambda)\{i(\pi / 4-\delta / 2)+\nu\})} \psi\left(e^{i(\pi / 2-\delta)+2 y}\right)\right. \\
& -\frac{1}{2}\left(e^{-\lambda\{i(\pi / 4-\delta / 2)+y\}}+e^{-(1-\lambda)\{i(\pi / 4-\delta / 2)+\nu\}}\right)
\end{aligned}
$$

are Fourier transforms. We now use formula (10.7.1), [2, p. 223], i.e.

$$
\begin{aligned}
\int_{-\infty}^{\infty}\left|\int_{t}^{t+H} F(u) d u\right|^{2} d t \leqq & 2 H^{2} \int_{0}^{1 / H}|f(y)|^{2} d y \\
& +8 \int_{1 / H}^{\infty}|f(y)|^{2} y^{-2} d y,
\end{aligned}
$$

where $H \geqq 1$ is a constant to be chosen later. Letting $y=\log x$ and $G=e^{1 / H}$, we see that (2.1) yields

$$
\begin{aligned}
\int_{-\infty}^{\infty}\left|\int_{t}^{t+H} F(u) d u\right|^{2} d t & \\
= & O\left\{H^{2} \int_{1}^{G}\left|\psi\left(e^{i(\pi / 2-\delta)} x^{2}\right)\right|^{2} d x\right\} \\
& +O\left\{\int_{G}^{\infty}\left|\psi\left(e^{i(\pi / 2-\delta)} x^{2}\right)\right|^{2} \frac{x^{1-2 \lambda}}{\log ^{2} x} d x\right\}+O(H) .
\end{aligned}
$$

The first integral on the right side of $(2.2)$ is estimated in [2, p. $224]$, and is $O\left(H \delta^{-1 / 2}\right)$ as $\delta$ tends to 0 .

To estimate the second we write

$$
\begin{aligned}
& \left|\psi\left(e^{i(\pi / 2-\delta)} x^{2}\right)\right|^{2} \\
& =\sum_{n=1}^{\infty} \exp \left[-2 n^{2} \pi x^{2} \sin \delta\right] \\
& \quad+\sum_{\substack{m=1 \\
m \neq n}}^{\infty} \sum_{n=1}^{\infty} \exp \left[-\left(m^{2}+n^{2}\right) \pi x^{2} \sin \delta+i\left(m^{2}-n^{2}\right) \pi x^{2} \cos \delta\right] .
\end{aligned}
$$

To examine the contribution of the first sum on the right side of (2.3), divide the interval $(G, \infty)$ into the subintervals $\left(G, \delta^{-1 / 2}\right)$ and $\left(\delta^{-1 / 2}, \infty\right)$ and let the corresponding integrals be denoted by $I_{1}$ and $I_{2}$, respectively. The sum under consideration is $O\left(x^{-1} \delta^{-1 / 2}\right)$, and so 


$$
\begin{aligned}
I_{1} & =O\left(\delta^{-1 / 2} \int_{G}^{\delta^{-1 / 2}} \frac{x^{1-2 \lambda}}{x \log ^{2} x} d x\right) \\
& =O\left(H \delta^{\lambda-1}\right),
\end{aligned}
$$

upon an integration by parts. The sum is also $O\left(\exp \left(-2 \pi x^{2} \delta\right)\right)$ for $x^{2} \delta \geqq 1$, and so

$$
\begin{aligned}
I_{2} & =O\left(\int_{\delta^{-1 / 2}}^{\infty} \frac{x^{1-2 \lambda}}{\log ^{2} x} \exp \left(-2 \pi x^{2} \delta\right) d x\right) \\
& =O\left(\delta^{\lambda} \int_{\delta^{-1 / 2}}^{\infty} x \exp \left(-2 \pi x^{2} \delta\right) d x\right) \\
& =O\left(\delta^{\lambda-1}\right) .
\end{aligned}
$$

The contribution of the second term on the right side of (2.3) is $O\left(H \delta^{-1 / 2}\right)$ by the same argument as that in $[2$, p. 224].

Letting $\delta=1 / T$ and

$$
I=\int_{t}^{t+H} \operatorname{Re} R(\lambda+i u) e^{(\pi / 4-1 / T) u} d u
$$

we have shown that

$$
\int_{T}^{2 T}|I|^{2} d t=O\left(H T^{1-\lambda}\right) .
$$

From the functional equation for $\zeta(s)$,

$$
\begin{aligned}
\operatorname{Re} R(\lambda+i t) & =\frac{1}{2}\{R(\lambda+i t)+R(\lambda-i t)\} \\
& =\frac{1}{2}\{R(\lambda+i t)+R(1-\lambda+i t)\} .
\end{aligned}
$$

Using the above and Stirling's formula for $\Gamma(\sigma+i t)$, we find that $|\operatorname{Re} R(\lambda+i t)| \geqq e^{-\pi t / 4}\left|K_{1} t^{(\lambda-1) / 2} \zeta(\lambda+i t)+K_{2} t^{-\lambda / 2} \zeta(1-\lambda+i t)\right|$. Thus, if $T \leqq t \leqq 2 T$,

$$
\begin{aligned}
J & =\int_{t}^{t+H}|\operatorname{Re} R(\lambda+i u)| e^{(\pi / 4-1 / T) u} d u \\
& \geqq T^{(\lambda-1) / 2} \int_{t}^{t+H}\left|K_{1} \zeta(\lambda+i u)+K_{2} u^{1 / 2-\lambda} \zeta(1-\lambda+i u)\right| d u \\
& \geqq T^{(\lambda-1) / 2}\left|\int_{t}^{t+H}\left\{K_{1} \zeta(\lambda+i u)+K_{2} u^{1 / 2-\lambda} \zeta(1-\lambda+i u)\right\} d u\right| .
\end{aligned}
$$


Using a simple approximation for $\zeta(s)[2$, p. 67], we have

$$
\begin{aligned}
T^{(1-\lambda) / 2} \geqq & \mid \int_{t}^{t+H}\left\{K_{1}\left(\sum_{n \leqq A T} n^{-\lambda-i u}+O\left(T^{-\lambda}\right)\right)\right. \\
& \left.\quad+K_{2} u^{1 / 2-\lambda}\left(\sum_{n \leqq A T} n^{\lambda-1-i u}+O\left(T^{\lambda-1}\right)\right)\right\} d u \mid \\
\geqq & \left(A_{1}+A_{2} T^{1 / 2-\lambda}\right) H+O\left\{\left|\int_{t}^{t+H} \sum_{2 \leq n \leqq A T} n^{-\lambda-i u} d u\right|\right\} \\
& +O\left\{\left|\int_{t}^{t+H} u^{1 / 2-\lambda} \sum_{2 \leqq n \leqq A T} n^{\lambda-1-i u} d u\right|\right\}+O\left(H T^{-\lambda}\right) .
\end{aligned}
$$

Employing the second mean value theorem for integrals to the second integral on the right side, we have

$T^{(1-\lambda) / 2} J \geqq A_{1} T^{1 / 2-\lambda} H+O\left(H T^{\llcorner\lambda}\right)$

$$
\begin{aligned}
& +O\left\{\left|\sum_{2 \leqq n \leqq A T}\left(1 / n^{\lambda+i(t+H)} \log n-1 / n^{\lambda+i t} \log n\right)\right|\right\} \\
& +O\left\{T^{1 / 2-\lambda}\left|\sum_{2 \leqq n \leqq A T}\left(1 / n^{1-\lambda+i(t+H)} \log n-1 / n^{1-\lambda+i \tau} \log n\right)\right|\right\} \\
& \geqq A_{1} T^{1 / 2-\lambda} H+\Psi,
\end{aligned}
$$

say, where $t<\tau<t+H$.

We show next that

$$
\int_{T}^{2 T}|\Psi|^{2} d t=O\left(T^{2-2 \lambda}\right) .
$$

Clearly, it is sufficient to examine

$$
\begin{aligned}
& \int_{T}^{2 T}\left|\sum_{2 \leqq n \leqq A T} 1 / n^{\lambda+i t} \log n\right|^{2} d t \\
& =\int_{T}^{2 T} \sum_{2 \leqq m \leqq A T} 1 / m^{\lambda+i t} \log m \sum_{2 \leqq n \leqq A T} 1 / n^{\lambda-i t} \log n d t \\
& =T \sum_{2 \leqq n \leqq A T} 1 / n^{2 \lambda} \log ^{2} n+\sum_{\substack{2 \leqq m, n \leqq A T \\
m \neq n}} \sum_{\substack{2 T\\
}}\left(1 /(m n)^{\lambda} \log m \log n\right) \int_{T}^{2 T}(n / m)^{i t} d t \\
& =O\left(T^{2-2 \lambda}\right)+O\left(\sum_{2 \leqq m<n \leqq A T} 1 /(m n)^{\lambda} \log m \log n \log n / m\right) .
\end{aligned}
$$

This last sum is estimated in exactly the same manner as (7.2.1), 
[2, p. 116], and is $O\left(T^{2-2 \lambda}\right)$. Replacing $\lambda$ by $1-\lambda$ in the above calculation, we find

$$
\int_{T}^{2 T}\left|\sum_{2 \unlhd n \leqq A T} 1 / n^{1-\lambda+i t}\right|^{2} d t=O\left(T^{2 \lambda}\right) .
$$

Thus, we have proved (2.6).

Now let $S$ denote the subset of $(T, 2 T)$ where $I=J$. Thus,

$$
\int_{S}|I| d t=\int_{S} J d t
$$

From (2.4),

$$
\begin{aligned}
\int_{S}|I| d t & \leqq \int_{T}^{2 T}|I| d t \leqq\left\{T \int_{T}^{2 T}|I|^{2} d t\right\}^{1 / 2} \\
& \leqq A_{2} H^{1 / 2} T^{1-\lambda / 2} .
\end{aligned}
$$

Also, from (2.5) and (2.6),

$$
\begin{aligned}
\int_{S} J d t & \geqq T^{(\lambda-1) / 2} \int_{S}\left(A_{1} T^{1 / 2-\lambda} H+\Psi\right) d t \\
& \geqq A_{1} H T^{-\lambda / 2} m(S)-T^{(\lambda-1) / 2} \int_{T}^{2 T}|\Psi| d t \\
& \geqq A_{1} H T^{-\lambda / 2} m(S)-T^{(\lambda-1) / 2}\left\{T \int_{T}^{2 T}|\Psi|^{2} d t\right\}^{1 / 2} \\
& \geqq A_{1} H T^{-\lambda / 2} m(S)-A T^{1-\lambda / 2},
\end{aligned}
$$

where $m(S)$ denotes the measure of $S$. Combining the above with (2.7), we find that

$$
A_{1} H T^{-\lambda / 2} m(S) \leqq A T^{1-\lambda / 2}+A_{2} H^{1 / 2} T^{1-\lambda / 2},
$$

or

$$
m(S) \leqq A H^{-1 / 2} T .
$$

Divide $(T, 2 T)$ into $[T / 2 H]$ pairs of abutting intervals $j_{1}, j_{2}$, each, except for possibly the last $j_{2}$, of length $H$. Then, if $j_{1}$ does not contain entirely points of $S$, either $j_{1}$ or $j_{2}$ contains a zero of $\operatorname{Re} R(\lambda+i t)$. Thus, if there are $\nu j_{1}$-intervals containing only points of $S, \nu H \leqq m(S)$, and $\operatorname{Re} R(\lambda+i t)$ has at least

$$
[T / 2 H]-\nu>T / 3 H-A T / H^{3 / 2}>T / 4 H
$$

zeros if $H$ is large enough. 
To prove (1.2) we merely observe that

$$
i \operatorname{Im} R(\lambda+i t)=\frac{1}{2}\{R(\lambda+i t)-R(1-\lambda+i t)\},
$$

and then proceed as before.

\section{REFERENCES}

1. B. Berlowitz, Extensions of a theorem of Hardy, Acta Arith. 14 (1968), 203-207.

2. E. C. Titchmarsh, The theory of the Riemann zeta-function, Clarendon Press, Oxford, 1951.

UNIVERSITY OF ILLINOIS 